\title{
Quantification of Surgical Blood Loss
}

\author{
MARCEL H. LEE, DVM, BRITT T. INGVERTSEN, DVM, JOLLE KIRPENSTEIJN, DVM, PhD, Diplomate ACVs \& ECVS, \\ ASGER L. JENSEN, DVM, DrVetSci, Diplomate ECVCP, and \\ ANNEMARIE T. KRISTENSEN, DVM, PhD, Diplomate ACVIM-SA \& ECVIM-CA
}

\begin{abstract}
Objective-To compare gravimetric and colorimetric methods of quantifying surgical blood loss, and to determine if there is a correlation between preoperative hemostatic tests (buccal mucosa bleeding time [BMBT] and intraoperative blood loss).

Study Design-Prospective clinical study.

Animals-Dogs $(\mathrm{n}=15)$ admitted for cutaneous tumor excision, orthopedic procedure, or exploratory laparotomy.

Methods-Intraoperative blood loss was quantified by measuring irrigation fluid and weighing surgical sponges used for blood and fluid collection during surgery. Results of gravimetric measurements were then correlated to blood loss quantified using spectrophotometric analysis of hemoglobin $(\mathrm{Hb})$ content. Hemostatic variables including BMBT were measured before surgery and compared with the calculated amount of blood loss.

Results-Blood loss quantified by gravimetric measurement showed a significant correlation with colorimetric determination of $\mathrm{Hb}$ content in surgical sponges and collected irrigation fluid $(\mathrm{r}=0.93$, $P<.0001)$. BMBT correlated weakly but significantly with intraoperative blood loss $(\mathrm{r}=0.56$, $P<.05)$.

Conclusions-Quantifying intraoperative blood loss using spectrophotometric $\mathrm{Hb}$ analysis accurately assessed the amount of blood loss; however, it is a time-consuming procedure, primarily applicable as a research tool. Gravimetric evaluation of intraoperative blood loss was found to be an accurate method, which can be recommended for use in a clinical setting.

Clinical Relevance-Estimation of blood loss using a gravimetric method is accurate and applicable in the clinical setting and provides surgeons with a simple and objective tool to evaluate intraoperative blood loss.

(C) Copyright 2006 by The American College of Veterinary Surgeons
\end{abstract}

\section{INTRODUCTION}

$\mathrm{E}$ STIMATION OF intraoperative blood loss is an integral part of any surgical procedure. Quantifying blood loss during a surgical procedure, supplemented with other variables such as blood pressure and tissue perfusion, is essential in the ongoing evaluation of a patient's condition and to assess and modify intra and postoperative management. Subjective, gravimetric, volumetric, radiometric, dilutional, and colorimetric methods have been used to estimate surgical blood loss in humans, ${ }^{1-3}$ but to our knowledge there are no veterinary studies that have evaluated quantitative methods for estimating intraoperative blood loss. Colorimetric measurement is currently considered to be the gold standard ${ }^{3}$; however, it is time consuming, tedious, and not practical in a clinical setting.

Many surgical procedures entail risk of acute intraoperative blood loss. ${ }^{4}$ Preoperative screening of the hemostatic system and identification of patients with increased risk of intraoperative bleeding is therefore important. Use of preoperative hemostatic variables including buccal

From The Small Animal Veterinary Teaching Hospital, Department of Small Animal Clinical Sciences, The Royal Veterinary and Agricultural University, Frederiksberg, Denmark.

Address reprint requests to Dr. Marcel H. Lee, DVM, The Small Animal Veterinary Teaching Hospital, Department of Small Animal Clinical Sciences, The Royal Veterinary and Agricultural University, Dyrlaegevej 16, 1870 Frederiksberg C, Denmark.

E-mail: lee@dsr.kvl.dk.

Submitted September 2005; Accepted January 2006

(C) Copyright 2006 by The American College of Veterinary Surgeons

0161-3499/06

doi:10.1111/j.1532-950X.2006.00162.x 
mucosal bleeding time (BMBT) is controversial because few veterinary reports concerning preoperative screening have been published. ${ }^{5-8}$ Only 1 of these reports correlates the findings with total intraoperative blood loss. ${ }^{6}$

Our objectives were to compare gravimetric and colorimetric methods of quantifying intraoperative blood loss in dogs during 3 different surgical procedures, and to compare the quantified intraoperative blood loss with preoperative hemostatic variables including BMBT to facilitate prediction of intraoperative blood loss.

\section{MATERIALS AND METHODS}

\section{Dogs}

Fifteen dogs (Table 1) admitted for 1 of 3 surgical procedures (exploratory laparotomy, orthopedic procedure, cutaneous tumor excision) between July and October 2001 were studied. The mean age of the dogs (11 females, 4 males) was 6.1 years (range, 4 months-12 years) and mean weight was $28.7 \mathrm{~kg}$ (range, 9-52 kg). Five dogs were enrolled for each procedure, performed by 1 of 3 different staff surgeons.

\section{Diagnostic Evaluation}

A complete physical examination was performed on each dog. Blood samples were collected from the jugular vein for evaluation of preoperative hemostatic variables. The first $2 \mathrm{~mL}$ of blood was discarded, after which 2 blood samples were collected (Vacuette 9NL Coagulation Sodium Citrate 3, $2 \%$, Greiner Bio-one, Kremsmünster, Austria) and then centrifuged at $2,630 \times \mathrm{g}$. for 5 minutes (Biofuge A, Heraeus Sepatech GmbH, Germany) within 1 hour of collection. The plasma was separated, aliquoted, and stored at $-80^{\circ} \mathrm{C}$. Co- agulation analysis for prothrombin time (PT), activated partial thromboplastin time (APTT), and fibrinogen was conducted using an ACL 9000 (Instrumentation Laboratories, Barcelona, Spain) while D-dimer was analyzed by the use of an immunoassay test kit (Nyco-Card D-dimer, Axis-Shield PoC AS, Oslo, Norway). All coagulation analyses were performed in 1 batch, within 6 months of freezing.

\section{Anesthetic Protocol}

Dogs were premedicated with diazepam $(0.4 \mathrm{mg} / \mathrm{kg}$ intramuscularly [IM]) and methadone $(0.3 \mathrm{mg} / \mathrm{kg}$ IM) and then induced with propofol ( $4 \mathrm{mg} / \mathrm{kg}$ intravenously [IV]) and maintained on isoflurane in oxygen. Blood pressure was monitored (DinamapTM Veterinary Blood Pressure Monitor 8300, Crittikon, FL) every 10 minutes. Fluid therapy with acetated Ringers (Ringer Acetat, Fresenius Kabi, Oslo, Norway) was administered during all surgical procedures at a rate of $10 \mathrm{~mL} /$ $\mathrm{kg} / \mathrm{h} \mathrm{IV}$.

Analgesia was provided on an individual basis but all dogs were administered preoperative methadone $(0.3 \mathrm{mg} / \mathrm{kg} \mathrm{IM})$ and a single postoperative dose of carprofen $(4 \mathrm{mg} / \mathrm{kg}$ IV) followed by carprofen $(2 \mathrm{mg} / \mathrm{kg}$ orally) twice daily for another 5 days. If deemed necessary, intraoperative pain management was achieved with fentanyl $(2 \mathrm{mcg} / \mathrm{kg}$ IV every 30 minutes during surgery).

\section{Evaluation of BMBT}

BMBT was measured under anesthesia before surgery started, using a double blade (Simplate II R, Oregano Teknika Corp., Durham, NC). ${ }^{6}$ Reported BMBT was calculated as the mean of the 2 bleeding times resulting from the double-blade incisions; all BMBT measurements were performed by the same person.

Table 1. Signalment of Patients, Blood Loss, and Hemostatic Data

\begin{tabular}{|c|c|c|c|c|c|c|c|c|c|c|c|c|}
\hline Patient* & Sex & $\begin{array}{c}\text { Age } \\
\text { (Months) }\end{array}$ & $\begin{array}{c}\text { Weight } \\
(\mathrm{kg})\end{array}$ & Breed & $\begin{array}{c}\text { Hemoglobin } \\
\text { Loss } \\
(\mathrm{nmol} / \mathrm{kg})\end{array}$ & $\begin{array}{c}\text { Blood } \\
\text { Loss } \\
(\mathrm{g} / \mathrm{kg} \mathrm{BW}) \\
\end{array}$ & $\begin{array}{c}\text { APTT } \\
\text { (seconds) }\end{array}$ & $\begin{array}{c}\text { PT } \\
\text { (seconds) }\end{array}$ & $\begin{array}{c}\text { Fibrinogen } \\
(\mathrm{g} / \mathrm{L})\end{array}$ & D-dimer & $\begin{array}{c}\text { BMBT } \\
\text { (seconds) }\end{array}$ & $\begin{array}{c}\text { Mean Blood } \\
\text { Pressure } \\
(\mathrm{mmHg}) \\
\end{array}$ \\
\hline 1 & $\mathrm{~F}$ & 40 & 32 & Mixed breed & 18,272 & 1.43 & 33.2 & 5.3 & 1.59 & 0.4 & 134 & 82 \\
\hline 2 & $\mathrm{~F}$ & 72 & 27.5 & Mixed breed & 29,773 & 4.87 & 11.7 & 6.4 & 0.99 & 0.8 & 299 & 75 \\
\hline 3 & $\mathrm{~F}$ & 91 & 35 & Golden Retriever & 13,364 & 1.57 & 28.0 & 5.5 & 1.49 & 0.3 & 221 & 70 \\
\hline 4 & $\mathrm{~F}$ & 24 & 30.5 & Alsatian & 13,104 & 1.31 & 10.4 & 5.8 & 1.10 & 0.1 & 173 & 75 \\
\hline 5 & $\mathrm{~F}$ & 60 & 9 & Mixed breed & 4565 & -0.22 & 10.4 & 6.0 & 1.27 & 0.1 & 108 & 55 \\
\hline 6 & $\mathrm{~F}$ & 114 & 37.5 & American Bulldog & 2533 & 0.29 & 10.4 & 6.1 & 2.11 & 0.5 & 160 & 80 \\
\hline 7 & $\mathrm{~F}$ & 126 & 26.6 & Pointer & 14,801 & 1.31 & 10.4 & 5.8 & 4.59 & 0.4 & 134 & 73 \\
\hline 8 & $\mathrm{~F}$ & 81 & 15.6 & Mixed breed & 31,514 & 4.87 & 40.2 & 5.6 & 2.80 & 0.3 & 111 & 78 \\
\hline 9 & M & 110 & 52 & Alsatian mix & 4607 & 0.90 & 10.7 & 5.7 & 1.82 & 0.3 & 146 & 74 \\
\hline 10 & $\mathrm{~F}$ & 132 & 23 & Mixed breed & 20,150 & 2.09 & 17.0 & 5.7 & 2.47 & 0.1 & 163 & 74 \\
\hline 11 & M & 105 & 42 & Alsatian mix & 14,353 & 0.74 & 10.4 & 6.0 & 1.14 & 0.2 & 138 & 81 \\
\hline 12 & $\mathrm{~F}$ & 32 & 32.8 & Mixed breed & 12,913 & -0.21 & 10.4 & 6.0 & 1.17 & 0.4 & 187 & 68 \\
\hline 13 & $\mathrm{~F}$ & 35 & 41 & Berner Sennen & 20,049 & 2.82 & 10.4 & 5.2 & 3.19 & 0.5 & 203 & 74 \\
\hline 14 & $\mathrm{M}$ & 78 & 31.5 & Golden Retriever & 65,765 & 8.41 & 40.0 & 5.3 & 2.22 & 0.3 & 204 & 80 \\
\hline 15 & M & 4 & 14.5 & Mixed breed & 54,543 & 6.62 & 14.2 & 5.9 & 12.50 & 0.5 & 330 & 72 \\
\hline
\end{tabular}

*Patients 1-5, laparotomy; 6-10, orthopedic procedure; 11-15, excision of cutaneous tumors.

BW, body weight; APTT, activated partial thromboplastin time; PT, prothrombin time. 


\section{Evaluation of Intraoperative Blood Loss}

Before surgery, a predetermined volume of sterile saline $(0.9 \% \mathrm{NaCl})$ solution for intraoperative usage (moistening of gauze sponges, etc.) was recorded and handed to the surgeon in a sterile bowl. Surgical sponges (surgical swabs X-ray detectable $10 \times 60 \mathrm{~cm}$, Klinidrape, Mölnlycke Health Care A/S, Allerød, Denmark) were weighed and all intraoperative blood loss around the surgical site was collected with moistened sponges and either transferred to the bowl of sterile saline solution or stored on a sterile plastic drape. To minimize loss of saline solution the surgeon expelled as much fluid from the sponges as possible before transferring the sponges from the instrument table to the surgical site.

Immediately after surgery, drapes (Buster Polyethylene $120 \times 180 \mathrm{~cm}$, Jørgen Kruuse A/S, Marslev, Denmark), instruments, surgical gowns, and surgical gloves used were cleaned of blood using moistened sponges, and these sponges together with all the sponges used intraoperatively were collected, counted, weighed, and stored at $-20^{\circ} \mathrm{C}$. The bloody saline solution remaining in the surgeon's bowl was measured immediately after surgery and 2 samples (each $1 \mathrm{~mL}$ ) were obtained and stored at $-20^{\circ} \mathrm{C}$. Intraoperative blood loss was determined as the weight difference between the sterile saline solution and gauze sponges pre and postoperatively.

\section{Hemoglobin Extraction Technique}

Saved gauze sponges were thawed to room temperature immediately before chemical testing. Sponges were soaked in $500 \mathrm{~mL}$ hemoglobin $(\mathrm{Hb})$ reagent $(\mathrm{PK}$ Hemoglobin Reagent Cyanide Free, Bie \& Bernsten A/S, Rødovre, Denmark) until all macroscopic traces of blood were dissolved, and then sponges were wrung out thoroughly and discarded. The total volume of solution was measured and 3 samples (each $1 \mathrm{~mL}$ ) were obtained and stored at $-20^{\circ} \mathrm{C}$. These samples were subsequently thawed and $2 \mathrm{~mL} \mathrm{Hb}$ reagent was added to each sample before renewed storage at $-20^{\circ} \mathrm{C}$. $\mathrm{Hb}$ reagent enables spectrophotometric measurement of $\mathrm{Hb}$ concentration by production of a stable reaction product of $\mathrm{Hb}$, heme, and all $\mathrm{Hb}$ types. $\mathrm{Hb}$ concentration in samples of blood soaked saline solution and $\mathrm{Hb}$ extracted samples from the surgical sponges were determined spectrophotometrically (SpectraMAX ${ }^{\mathrm{sm}}$, Biotec Line A/S, Slangerup, Denmark) using human $\mathrm{Hb}$ standards (JT Barker, 3074, Bie \& Berntsen A/S).

\section{Statistical Analysis}

Statistical analysis was performed using software (SAS Institute Inc., Cary, NC). Correlations of blood loss quantified by the colorimetric and the gravimetric methods were compared statistically by nonparametric Spearman's rank correlation test and scatter plot. Correlations between surgical blood loss and continuous hemostatic variables (BMBT, PT, APTT, fibrinogen, and D-dimer), duration of surgery, and age were determined by analysis of variance (ANOVA; PROC MIXED, SAS Institute) whereas differences in blood loss within the variables of sex, surgical procedure, and surgeon was performed using a Kruskal-Wallis rank sum analysis. A $P$-value $<.05$ was considered significant.

\section{RESULTS}

\section{Evaluation of Intraoperative Blood Loss}

Colorimetric quantification of blood loss using spectrophotometric $\mathrm{Hb}$ analysis was significantly correlated to the gravimetric method $(\mathrm{r}=0.93, P<.0001$; Table 1 ; Fig 1). None of the dogs lost $>10 \%$ of their estimated blood volume except dog 14 (11\% loss). Surgical procedure and surgeon did not significantly influence the volume of intraoperative blood loss (Table 2). No significant correlation was observed between intraoperative blood loss and any of the coagulation variables (APTT, PT, fibrinogen, D-dimer), mean blood pressure during surgery, or duration of surgery, although there was a trend toward significance for APTT $(P=.06)$ and duration of surgery $(P=.09$; Table 1$)$. Five dogs had a prolonged APTT. No significant difference was found between the descriptive variables of patient age, weight, sex, and volume of intraoperative blood loss. Volume of intraoperative blood loss was significantly correlated to BMBT $(\mathrm{r}=0.56 ; P<.05 ;$ Fig 2$)$. Two dogs had prolonged BMBT with concurrent normal coagulation variables and greater than average blood loss whereas 2 dogs had normal BMBT and greater than average blood loss. None of the dogs had excessive intraoperative bleeding or complications arising from bleeding. None of the dogs subsequently had a history or clinical signs of postoperative bleeding.

\section{DISCUSSION}

We found a highly significant correlation between colorimetric and gravimetric methods of quantifying in-

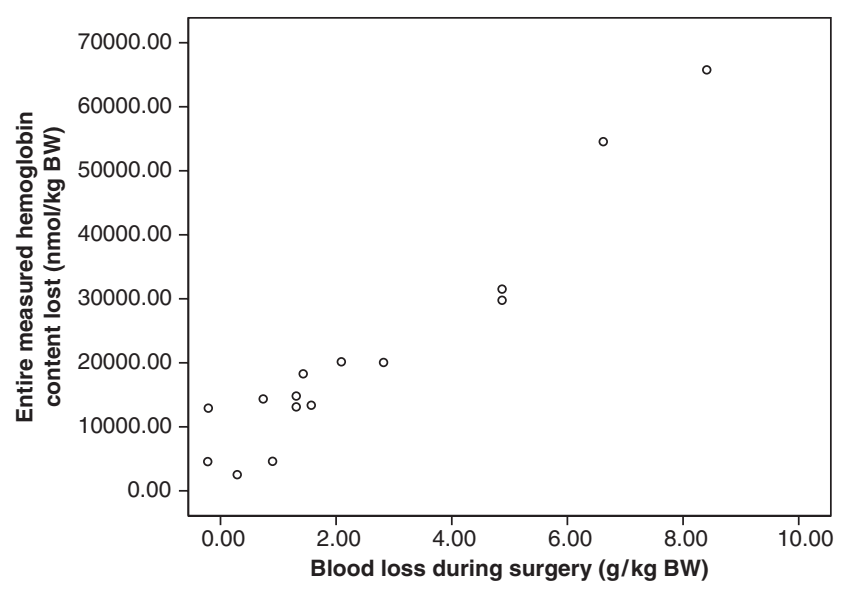

Fig 1. Correlation between colorimetric and gravimetric methods of evaluating intraoperative blood loss. 
Table 2. Influence of Surgical Procedure on Blood Loss and Surgical Time and the Influence of Surgeon on Blood Loss and Surgical Time

\begin{tabular}{|c|c|c|c|c|}
\hline & $\mathrm{N}$ & Mean & SD & SE \\
\hline \multicolumn{5}{|l|}{ By procedure } \\
\hline \multicolumn{5}{|l|}{$\begin{array}{l}\text { Hemoglobin content } \\
(\mathrm{nmol} / \mathrm{kg} \mathrm{BW})\end{array}$} \\
\hline Laparotomy & 5 & $15,815.67$ & 9230.18 & 4127.87 \\
\hline Tumor extirpation & 5 & $14,719.03$ & 11859.05 & 5303.53 \\
\hline Orthopedic procedure & 5 & $33,524.86$ & $24,775.09$ & $11,079.76$ \\
\hline Total & 15 & $21,353.19$ & 17873.93 & 4615.03 \\
\hline \multicolumn{5}{|l|}{$\begin{array}{l}\text { Blood loss during surgery } \\
(\mathrm{g} / \mathrm{kg} \mathrm{BW})\end{array}$} \\
\hline Laparotomy & 5 & 1.79 & 1.87 & 0.83 \\
\hline Tumor extirpation & 5 & 1.89 & 1.79 & 0.80 \\
\hline Orthopedic procedure & 5 & 3.67 & 3.73 & 1.67 \\
\hline Total & 15 & 2.45 & 2.58 & 0.67 \\
\hline \multicolumn{5}{|l|}{ Surgical time (minutes) } \\
\hline Laparotomy & 5 & 129.00 & 32.48 & 14.53 \\
\hline Tumor extirpation & 5 & 114.00 & 44.64 & 19.96 \\
\hline Orthopedic procedure & 5 & 154.00 & 95.22 & 42.59 \\
\hline Total & 15 & 132.33 & 61.26 & 15.82 \\
\hline \multicolumn{5}{|l|}{ By surgeon } \\
\hline \multicolumn{5}{|l|}{$\begin{array}{l}\text { Hemoglobin content } \\
(\mathrm{nmol} / \mathrm{kg} \mathrm{BW})\end{array}$} \\
\hline Surgeon 1 & 2 & $36,407.76$ & $25,647.48$ & $18,135.51$ \\
\hline Surgeon 2 & 5 & $28,570.80$ & $21,822.06$ & 9759.12 \\
\hline Surgeon 3 & 8 & $13,078.54$ & 9625.65 & 3403.18 \\
\hline Total & 15 & $21,353.19$ & $17,873.93$ & 4615.03 \\
\hline \multicolumn{5}{|l|}{$\begin{array}{l}\text { Blood loss during surgery } \\
(\mathrm{g} / \mathrm{kg} \mathrm{BW})\end{array}$} \\
\hline Surgeon 1 & 2 & 4.03 & 3.67 & 2.60 \\
\hline Surgeon 2 & 5 & 3.33 & 3.45 & 1.54 \\
\hline Surgeon 3 & 8 & 1.52 & 1.54 & 0.54 \\
\hline Total & 15 & 2.45 & 2.58 & 0.67 \\
\hline \multicolumn{5}{|l|}{ Surgical time (minutes) } \\
\hline Surgeon 1 & 2 & 215.00 & 134.35 & 95.00 \\
\hline Surgeon 2 & 5 & 128.00 & 48.04 & 21.48 \\
\hline Surgeon 3 & 8 & 114.38 & 35.70 & 12.62 \\
\hline Total & 15 & 132.33 & 61.26 & 15.82 \\
\hline
\end{tabular}

traoperative blood loss. Thus the gravimetric method can be used to accurately quantify intraoperative blood loss in preference to the colorimetric method, which is time consuming and thus more suited as a research technique. Significant correlation was also found between surgical blood loss and BMBT whereas no significant correlation was found between blood loss and other preoperative hemostatic variables (APTT, PT, fibrinogen, and Ddimer). Data for the 15 dogs were pooled because no significant difference in volume of blood loss was observed despite different surgical procedures performed.

Although no veterinary reports are available, a human study evaluating gravimetric and colorimetric (accepted as the "gold standard") estimation of intraoperative blood loss found no significant correlation between these methods. ${ }^{3}$ Unfortunately, in that report the source of blood collection (operating room [OR] floor, OR table,

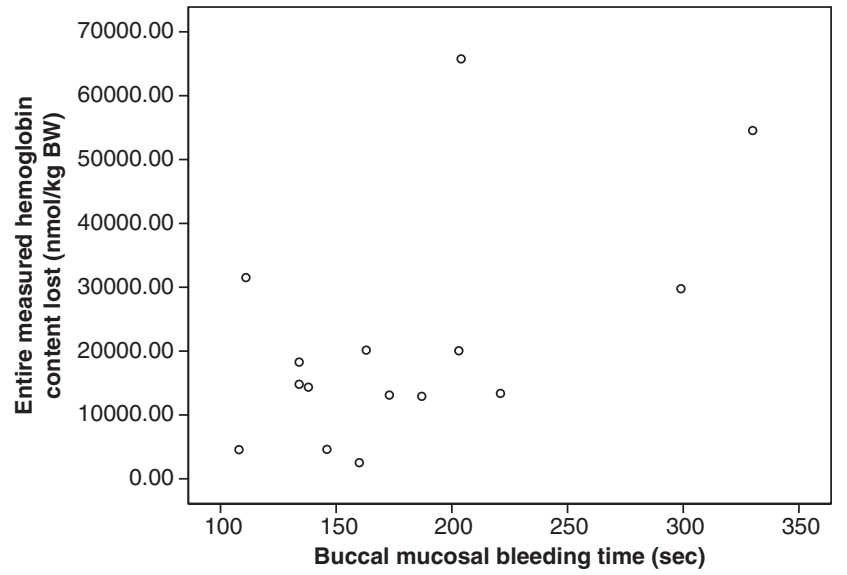

Fig 2. Correlation between preoperative buccal mucosa bleeding time and intraoperative blood loss.

drapes, etc.), method of sponge collection and storage, and accounting for sterile irrigation fluid contamination of the sponges was not reported. No information about bleeding tendencies or coagulation abnormalities in the patient population was provided and, further, the study correlated calculated blood loss $(\mathrm{mL})$ with weighed blood loss $(\mathrm{mL})$. The differences in study design may explain the conflicting results with our report.

Several human reports describe evaporation from surgical sponges as a flaw that discards gravimetric evaluation as a plausible means for reliable blood loss estimation. ${ }^{1,2,9}$ In our study, 2 dogs had a negative volume of intraoperative blood loss by gravimetric evaluation (Table 1). Evaporation may be a plausible explanation for these negative values. Gravimetric quantification of blood loss, by weighing sponges, may underestimate true blood loss. The colorimetric method should not be influenced by evaporation and because it was significantly correlated with the gravimetric method, we conclude that evaporation does not significantly influence estimation of total intraoperative blood loss. It is also reasonable to posit that evaporation was a constant factor or error in all 15 cases we studied.

It is imperative that unaccounted intraoperative blood loss to gloves, instruments, gowns, surgery drapes, surgery table, patient padding, and OR floor is kept to a minimum to ensure good correlation between colorimetric/gravimetric measurement and actual intraoperative blood loss. Our patients did not suffer from extensive intraoperative bleeding or blood loss, which meant that almost all intraoperative blood loss could be retrieved by meticulously cleaning instruments, gloves, gowns, and surgery drapes. Neither blood loss to the OR table or floor was observed. Given that none of the dogs lost $>11 \%$ of their estimated blood volume, no extensive intraoperative bleeding was encountered, and meticulous 
collection of intraoperative blood loss was conducted under strict adherence to a very detailed protocol, we feel confident that the amount of unaccounted blood loss was kept to an absolute minimum in this study and thereby eliminates an otherwise serious bias to the correlation.

The fact that there was a significant correlation between colorimetric and gravimetric evaluation of intraoperative blood loss could have an impact on future studies, because the gravimetric method is less time consuming and patient blood loss can be quickly determined postoperatively, allowing further corrective treatment to be quickly instituted.

A preoperative test that might reliably predict patients at risk for intraoperative blood loss would be valuable for perioperative patient management. Preoperative BMBT had a weak but significant correlation with intraoperative blood loss. This was surprising because BMBT is usually regarded as an unreliable and variable test with low positive predictive value in assessing the risk of intraoperative blood loss $^{10-12}$; however, our results corroborate another veterinary study. ${ }^{6}$ The experimental design of that study was quite different because the amount of intraoperative blood loss was subjectively estimated based on surgeon observation without direct quantification. Our results might also be biased because none of the dogs had excessive intraoperative blood loss or hemostatic disorders and our sample size was limited. To support a correlation between BMBT and intraoperative blood loss, a direct comparison between a population of dogs with normal and abnormal BMBT values subjected to different surgical procedures would have to be conducted with larger sample sizes.

We found no significant correlation between volume of intraoperative blood loss and preoperative coagulation variables (APTT, PT, fibrinogen, and D-dimer), despite 5 prolonged APTT measurements. Many factors, including stress, can influence the accuracy of coagulation profiles. ${ }^{13}$ To minimize falsely elevated coagulation values, the first vial of blood collected is discarded because tissue factor is immediately activated upon needle penetration of the vein. ${ }^{13}$ Vials must all be filled with the precise volume of blood to ensure the correct blood:citrate concentration. A common mistake is to collect too small a sample (loss of vacuum in the vial, time restriction, nervous dog, etc.), resulting in a reduced calcium availability and thereby prolonged fibrin production time manifested as a prolonged coagulation profile. ${ }^{14}$ Factors such as hemoconcentration, hemolysis, handling of blood samples, etc. are important to consider when evaluating the precision of coagulation analysis in dogs. ${ }^{15,16}$ Several authors conclude that APTT is prone to variations because of sensitivity of sampling and handling of the citrate stabilized blood as well as instability in relation to freezing. ${ }^{17-20}$ Therefore, preanalytic errors including formation of cryoprecipitate cannot be excluded as an explanation of the 5 prolonged APTT values we measured. Otherwise, our findings correspond with results from studies of humans. ${ }^{21-23}$

Summarily, we found a significant correlation between the "gold standard" colorimetric quantification of blood loss and a gravimetric method. Quantifying intraoperative blood loss can be performed objectively using a simple gravimetric method rather than the time-consuming colorimetric method. We also found a weak but significant correlation between elevated BMBT and increased intraoperative blood loss in certain procedures in dogs without hemostatic disorders. Further studies with different groups of dogs, with and without extensive intraoperative bleeding and/or hemostatic disorders, are needed to evaluate the association between BMBT, intraoperative blood loss, and complications associated with bleeding before general recommendations can be made.

\section{ACKNOWLEDGMENTS}

Birgitte Klitgaard, Hemostasis Pharmacology, Novo Nordisk A/S is thanked for expert technical assistance with the $\mathrm{Hb}$ measurements and Jens $\mathrm{S}$. Madsen, DVM, PhD for participation during collection of blood lost during orthopedic procedures.

\section{REFERENCES}

1. Budny PG, Regan PJ, Roberts AH: The estimation of blood loss during burns surgery. Burns 19:134-137, 1993

2. Flordal PA: Measurement of blood loss in clinical studies. Eur J Anaesthesiol 14(Suppl.): 35-37, 1997

3. Johar RS, Smith RP: Assessing gravimetric estimation of intraoperative blood loss. J Gynecol Surg 9:151-154, 1993

4. Wagner AE, Dunlop CI: Anesthetic and medical management of acute hemorrhage during surgery. J Am Vet Med Assoc 203:40-45, 1993

5. Brassard JA, Meyers KM: Evaluation of the buccal bleeding time and platelet glass bead retention as assays of hemostasis in the dog: the effects of acetylsalicylic acid, warfarin and von Willebrand factor deficiency. Thromb Haemost 65:191-195, 1991

6. Jergens AE, Turrentine MA, Kraus KH, et al: Buccal mucosa bleeding times of healthy dogs and of dogs in various pathologic states, including thrombocytopenia, uremia, and von Willebrand's disease. Am J Vet Res 48:1337-1342, 1987

7. Forsythe LT, Willis SE: Evaluating oral mucosa bleeding times in healthy dogs using a spring-loaded device. Can Vet J 30:344-345, 1989

8. Sato I, Anderson GA, Parry BW: An interobserver and intraobserver study of buccal mucosal bleeding time in Greyhounds. Res Vet Sci 68:41-45, 2000 
9. Gombotz H, Metzler H, List WF: Methods for reduction of perioperative bleeding. Br J Anaesth 81(Suppl. 1): 62-66, 1998

10. De Rossi SS, Glick M: Bleeding time: an unreliable predictor of clinical hemostasis. J Oral Maxillofac Surg 54: 1119-1120, 1996

11. De CR, Lanza M, Manca G, et al: Bleeding time and bleeding: an analysis of the relationship of the bleeding time test with parameters of surgical bleeding. Blood 84:3363-3370, 1994

12. Rodgers RP, Levin $\mathrm{J}$ : A critical reappraisal of the bleeding time. Semin Thromb Hemost 16:1-20, 1990

13. Green RA, Hammer AS: Hemostatic Disorders: Coagulopathies and Thrombosis, in Ettinger SJ, Feldman EC (eds) Textbook of Veterinary Internal Medicine, Vol. 2 (ed 4). Philadelphia, PA, Saunders, 1995, pp 1946-1964

14. Johnstone IB: The importance of accurate citrate to blood ratios in the collection of canine blood for hemostatic testing. Can Vet J 34:627-629, 1993

15. Moreno P, Ginel PJ: Effects of haemolysis, lipaemia and bilirubinaemia on prothrombin time, activated partial thromboplastin time and thrombin time in plasma samples from healthy dogs. Res Vet Sci 67:273-276, 1999

16. O'Brien SR, Sellers TS, Meyer DJ: Artifactual prolongation of the activated partial thromboplastin time associated with hemoconcentration in dogs. J Vet Intern Med 9: 169-170, 1995
17. Evans GO, Flynn RM: Activated partial thromboplastin time measurements in citrated canine plasma. J Comp Pathol 106:79-82, 1992

18. Ho $\mathrm{CH}$, Wu SY: The influence of time, temperature and packed cell on activated partial thromboplastin time and prothrombin time. Thromb Res 62:625-633, 1991

19. Peterson P, Gottfried EL: The effects of inaccurate blood sample volume on prothrombin time (PT) and activated partial thromboplastin time (aPTT). Thromb Haemost 47:101-103, 1982

20. Rao LV, Okorodudu AO, Petersen JR, et al: Stability of prothrombin time and activated partial thromboplastin time tests under different storage conditions. Clin Chim Acta 300:13-21, 2000

21. Asaf T, Reuveni H, Yermiahu T, et al: The need for routine pre-operative coagulation screening tests (prothrombin time PT/partial thromboplastin time PTT) for healthy children undergoing elective tonsillectomy and/or adenoidectomy. Int J Pediatr Otorhinolaryngol 61:217222, 2001

22. Suchman AL, Mushlin AI: How well does the activated partial thromboplastin time predict postoperative hemorrhage? JAMA 256:750-753, 1986

23. Velanovich V: The value of routine preoperative laboratory testing in predicting postoperative complications: a multivariate analysis. Surgery 109:236-243, 1991 